Calculus IN THE Bladder.-OPERATION.

G. P., a groom, twenty years of age, was admitted on the 25 th of $\mathbf{J u l y}$, nuder the care of Mr. Liston. Nine years ago he first found considerable dificulty in making water, and passed some blood with it. Shortly afterwards he voided a large quantity of gravel with his urine. With the exception of the passage of the blood, he has suffered from the above symptoms to the present time. In addition to these, he had also pain in the perineum, the urine stops suddenly, and he had pain in the bladder on using any exertion by which the body was shaken. General health always good. Complains now of pain across the lower region of the abdomen, and in the lumbar region; no pain at the end of the penis; urine stops suddenly during micturition. There is a thick sediment, but it is not ropy, in the urine after standing a short time. Great pain in the perineum. Two or three days after his admission, Mr. Liston performed the bilateral operation, which was done in the usual manner, and a middling sized, very rough, mulberry calculus was extracted; the patient went on well and is now about to leave the hospital.

TUMOUR OF THE HAND-continued from $\% .491$. July 20. J. B.'s hand is now completely healed. He has already experienced the value of the treatment employed, as he is now able to apply the limb to various uses.

\section{BRIDGEWATER INFIRMARY.}

\section{RESUSCITATION OF STILL-BORN CHILDREN.}

By Jonathan Toogood Esq., Surgeon to the Bridgewat er Infirmary.

CAscs of apparently still-born infants are very common. The attempts to restore life are frequently ill-directed, and not calculated to promote the object. It will be found that a very large proportion of chil. dren, apparently dead born, may be resuscitated, if proper means be resorted to and persevered in for a sufficient length of time; but the modes generally employed to restore life, such as immersing the infant in warm water, friction, and pouring stimulants down the throat, are not at all calculated to produce the effect intended, and if these means do not succeed after a short trial, all further attempts are generally abandoned. The plan I always adopt, which has never failed where the child was living during birth, is very simple, and only requires perseverance. The following cases, under circumstances by no means favourable, which have been selected from a great many more, will prove the success of the practice recommended.
Grace White, a very weakly woman, far advanced in consumption, was seized, in the morning, with uterine hæmorrhage, which continued slightly till the evening, when I saw her, and, whilst standing by her bed. side, the flooding increased with such vio. lence, that I thought it best to deliver her instantly; the child was still-born. As soon as I had removed it from the nother, and seen her safe from any immediate dan. ger, I placed a napkin over the child's mouth, and inflated its longs from my mouth, pressing out the air from the chest after. wards, and thus imitating natural respira. tion. After having continued this process for thirty-five minutes, the child made a very slight attempt to breathe, and the face became slightly suffused; by persevering ten minutes longer, the frec action of the lungs was established, and the child cried lustily.

The next case was that of a poor wonan, named Sarals Holmes, of the parish of Spaxton, who had been in labour a long time, with a presentation of the arm, and, as it was her first confinement, it became very difficult to tuin the child, particulaly as she was advanced in age, and the parts were very rigid. The child was still-born; but, by pursuing the same plan actively for three-quarters of an hour, animation was perfectly restored.

The next was a case of presentation of the funis, and as the labour was slow, the child was still-born, but recorered by the same means in half an hour.

The last case with which I shall trouble you, was such as to encourage the attempt at resuscitation under any circumstances; it was a case of twins, and the second child presented with the head, before which a considerable portion of the funis had descended. The delivery was extremely slow, from the general weakness of the woman, who had been for a long time in a bad state of health, and the child was born, apparently, quite dead. As the mother's situation was extremely critical, more than half an hour had elapsed before I could at. tend to the child, and, on inquiring, I found it had been wrapped in a cloth and placed on a chair in another room. I immediately made the attempt to restore it, and, by persevering steadily for twenty-five minutes, [ had the satisfaction to see symptoms of re. turning life ; and, in about fifteen minutes more, the child breathed freely.

Everything in this last case was unfavour. able to the restoration of the child; the mother's long-continued disease; the cir. cumstance of her having two children, and more particularly the delay which took place before any attempt was made, during. which time the child was exposed in a room without fire, in the winter, with a partial and very slight covering. I am warranted, by my own experience, in recom. 
mending the attempt to restore all still-born children who have been alive during the birth; and if the means of resuscitation, above mentioned, be actively employed, and steadily persevered in, $I$ believe the majority of cases will be successful. In all cases the restoration of a child is a most satisfactory circumstance, and, in some instances, of the greatest possible consequence. I have never found any thing necessary but the regular inflation of the lungs, which $I$ do with my own mouth in the way $I$ have described, and have generally observed the first symptom of returning life to be a tremulous motion of the respiratory organs; the child next makes a feeble attempt to inspire, and the colour of the face changes. The inflation should then be made quicker, and as the attempts to breathe increase, sal volatile, or brandy, rubbed over the palm of the hand, and held over the mouth during the inflation of air, will materially assist the recovery, and has a better effect than pouring stimulants into the stomach. A few smart slaps on the gluteal muscles will now generally complete the recovery.

It has always been my practice to expire as completely, and immediately to inhale as much air as possible, and this should be repeated frequently during the process of inflating the lungs.

\section{STRICTURE OF THE VAGINA.}

A woman, about forty, who had borne children, and whose labours had been slow and lingering, but not dangerous, considered herself again pregnant about six months after she had given birth to a child. The catamenia bad entirely ceased, and although she had no doubt of her situation she was remarked not to increase in size. At this time she had some discharge, and suspected she was going to miscarry, but as nothing more than dark-coloured blood passed, and the pains became very severe, attended with shivering and considerable fever ; she consulted a surgeon who had been accustomed to the practice of midwifery, who discovered, on examination, a circular contraction of the vagina, so complete as almost entirely to obliterate the canal, leaving an orifice scarcely large enough to admit the point of a probe, through which a dark-coloured and bjghly offensive fluid passed. The opening was gradually dilated, which allowed the escape of a very large quantity of putrid fluid, with immediate relief of all the symp. toms. The stricture was now forcibly dilated for seventeen days, when she was pronounced cured. About two months after this period she fell under my care, when I found the contraction so great that $I$ could not insert the point of my finger into it, without using much force and occasioning considerable pain. Having succeeded in effectually dilating the female urethra by the introduction of sponge tent, without the slightest pain, in two cases in which a female catheter had accidentally slipped into the bladder, $I$ determined to make trial of it in this case, and so easily effected it, that in the course of a few days, the stricture was completely dilated, and the canal apparently restored to its natural state. Two days after the removal of the sponge I had the mortification to find the contraction as great as ever, and believing that I had not kept up the dilatation long enough, I gradually in. troduced a very large piece of sponge, and allowed it to remain several days, but on removing it I again found that $I$ had gained nothing. As my patient was unwilling to submit to severer treatment, and fancied that her recovery was only protracted because the remedies had not been continued for a sufficient length of time, the same plan was persevered in for a month with no better success. The sponge tent was now introduced and removed with so much ease, that she was in the habit of doing it herself, when, on one occasion, the string broke, and a very large piece of sponge was left in the upper part of the vagina, the stricture closing below it. Several ineffectual attempts were made to remove it, which was at length effected by introducing a smaller tent into the orifice of the stricture, which admitted the blades of a pair of stone forceps, with which it was grasped and brought away. It was now evident that nothing but the actual division of the muscular fibres of the vagina, in many differeut places, and to a considerable extent, would succeed in obtaining a permanent cure. This practice was adopted, and a very large dilator made of cork, and covered with caoutchouc, introduced, but so great was the disposition of the parts to contract, even after the incisions which had been made so freely had healed, that it was necessary to wear the instrument for many months before the cure could be pronounced to be complete.

I have had frequent opportunities of examining this patient, who remains quite well, and it is now nearly two years since the operation was performed.

I found it extremely difficult to get an effectual dilator. Conmon rectum bougies, wax-candles, and such means as are usually employed, were extremely inconvenient, and failed; but the dilator which $\mathrm{Mr}$. Laurie made, under my direction, answered so well that I shou ld recommend it with confidence in all such cases. A strong silk ligature passed through and brought down by the sides of the cork, over which the choutchouc was stretched, was made into a loop, and to this a napkin was attached. This was worn without interruption of exercise or the natural functions: 